\title{
Nucleosynthesis in classical nova explosions and type I X-ray bursts
}

\author{
Anuj Parikh* \\ Dept. Física i Enginyeria Nuclear, Universitat Politècnica de Catalunya (UPC), and \\ Institut d'Estudis Espacials de Catalunya (IEEC) \\ C. Urgell 187, EUETIB, E-08036 Barcelona, Spain \\ E-mail: anuj.r.parikh@upc.edu
}

\begin{abstract}
Classical nova explosions and type I X-ray bursts are the most frequent types of thermonuclear stellar explosions in the Galaxy. Both phenomena arise from thermonuclear ignition in the envelopes of accreting compact objects in close binary star systems. Detailed observations of these events have stimulated numerous studies in theoretical astrophysics and experimental nuclear physics. We briefly discuss recent theoretical efforts to understand the nucleosynthesis in these explosions, as well as experimental efforts to measure nuclear reactions and masses whose uncertainties significantly affect model predictions.
\end{abstract}

XII International Symposium on Nuclei in the Cosmos

August 5-12, 2012

Cairns, Australia

\footnotetext{
* Speaker.
} 


\section{Introduction}

Since at least the second century CE, with the event now known as SN 185, astronomers have recorded the sudden appearance of bright, star-like phenomena in the night sky that gradually fade with time. The term nova was eventually used for all such events. It was only in the 1920s and 1930s, through dedicated studies examining the distances to different nova events (distinguishing those of Galactic origin from those at much greater distances) that the distinct terms supernova and nova were introduced to differentiate between the intrinsically brighter supernova events and the dimmer (though still spectacular) nova events. Several hundred Galactic novae have been discovered to date, with $\approx 5$ events discovered per year. They are characterized by peak luminosities of $10^{4}-10^{5} L_{\odot}$, light curves of $\approx$ days to months in duration, and mass ejection into the interstellar medium of $\approx 10^{-5}-10^{-4} M_{\odot}$ per event. The recurrence period for novae is expected to be $10^{4}$ $-10^{5}$ years, although rare recurrent novae (with periods on the order of decades) have also been observed. For reviews on classical nova explosions (CN) see, e.g., Refs. [1,2].

In contrast, type I X-ray bursts (XRBs) were only discovered roughly 40 years ago. They were recognized as qualitatively different phenomena from previously observed X-ray events in part by their fast rise times (on the order of $1 \mathrm{~s}$ ). Roughly 100 bursting systems have been identified in the Galaxy, with light curves of $10-100 \mathrm{~s}$ in duration, recurrence periods of $\approx$ hours to days and similar peak luminosities to those of classical novae. Calculations indicate that radiative winds generated during some bursts may eject material; studies are ongoing to examine the viability of detecting absorption features arising from this material. For reviews on aspects of type I X-ray bursts, see, e.g., Refs. [3-5].

\section{Models}

Both classical nova explosions and type I X-ray bursts arise from thermonuclear runaways within the accreted envelopes of compact objects in close binary systems, with orbital periods often less than $\approx 15$ hours. Generally in a classical nova, H-rich material is transferred from a low mass main sequence or red giant star onto the surface of a white dwarf star. In a type I Xray burst, a neutron star is envisaged with a similar low mass companion star; observations are consistent with the accretion of material enriched in $\mathrm{H}, \mathrm{He}$ or both. Typical accretion rates in these events range from $\approx 10^{-10}-10^{-9} M_{\odot} / \mathrm{yr}$, although the range and implications of different accretion rates (resulting in e.g., stable or marginally stable burning for an accreting neutron star [6]) are still under investigation. As accretion proceeds, the envelope is gradually compressed and becomes degenerate. The temperature of the envelope increases, creating conditions favorable to the ignition of the accreted fuel through nuclear reactions. These reactions, once initiated, drive further reactions, leading to the thermonuclear runaway and the corresponding explosion. Note that the degeneracy of the envelope is lifted as the temperature increases at early times. For example, for the accretion of material with $Z / A \approx 0.5$, degeneracy is lifted at $T \approx 30 \mathrm{MK}$ or $\approx 300 \mathrm{MK}$ for a white dwarf or neutron star envelope, respectively. The difference between $\mathrm{CN}$ and XRBs from the viewpoint of mass ejection arises from how in the latter case the necessary escape velocities are never achieved. Here, for XRBs, we focus on bursts in H-rich envelopes; see e.g., Refs. [7-10] for helium and carbon flashes. 
Pioneering calculations by Schatzman [11-13] for CN and by Woosley and Taam [14] and Maraschi and Cavaliere [15] for XRBs eventually led to numerous investigations of the effects on model predictions of changes to the accretion rate, composition of the accreted material, size of the compact object and the initial surface luminosity of the compact object. Indeed, such studies continue today [16-20]. Examination of the detailed nucleosynthesis during CN and XRBs required the use of large nuclear reaction networks; these were lacking in early models in which only reaction links necessary to roughly approximate the thermonuclear energy release were included due to computational limitations.

Modern predictions of nucleosynthesis in $\mathrm{CN}$ and XRBs do use large nuclear networks $(\approx 150$ isotopes for $\mathrm{CN}, \approx 600$ isotopes for XRBs) and generally rely upon either simple one-zone treatments or 1D hydrodynamic models. In the first of these frameworks, nuclear networks are evolved using temperature-density-time thermodynamic histories from a single layer of the envelope. These histories may be extracted directly from a hydrodynamic model (using, e.g., the layer that achieves the highest peak temperature [21]) or may be determined through semi-analytic models [22]. Such treatments neglect (or simplify) essentially hydrodynamic effects such as convection and the finite diffusion time for energy to escape from the accreted envelope, but are significantly faster than hydrodynamic studies. For this reason one-zone models have been adopted in studies requiring a large number of calculations, e.g., the examination of the effects of nuclear physics uncertainties on model predictions $[21,23]$. Studies to overcome some of the disadvantages of one-zone models, involving e.g., the processing of multiple zones in concert with some procedure to emulate the results of convective mixing, are under investigation.

Exciting results have recently been reported from multidimensional studies of CN [24] and XRBs [25]. For example, Casanova et al. [24], in a 3D CN simulation, successfully reproduce observed metallicity enhancements in nova ejecta through convective mixing at the core-envelope interface. Prior expanations had not been successful primarily because they had only been proposed and tested in the framework of 1D or 2D nova models. Reliable nucleosynthesis predictions are not yet available from such studies however, both due to the limited nuclear physics networks adopted, and more importantly, due to how an examination of the full explosion from the initial to final stages has not yet been made.

\section{Nucleosynthesis}

Currently, the most reliable predictions of nucleosynthesis and light curves in CN and XRBs arise from 1D spherically-symmetric hydrodynamic models [16, 17, 20, 26, 27]. As an example, Fig. 1 illustrates a (perhaps extreme) case of issues arising when comparing results obtained from one-zone and hydrodynamic models. The top panel shows final abundances at the end of an XRB from a full hydrodynamic model, and, using only the hottest shell of the same model in a one-zone treatment. The bottom panel compares the nuclear energy generation rates from both models for that same shell. Discrepancies are clear in the abundances, although similar trends are observed (e.g., in the higher relative abundances around the waiting points $A=60,64,68,72,76,80$ ). More significant disagreement is seen in the nuclear energy generation rates; this is related to how, in the hydrodynamic model, nuclear energy released within the ignition shell, deep inside the envelope, 
is convectively transported towards the surface. This effect, as well as the consequent effect on the predicted XRB light curve, cannot be self-consistently handled within one-zone treatments.

Because nucleosynthesis in classical nova explosions is normally restricted to nuclei near the valley of stability and to masses less than $A \approx 40$, many of the reaction rates involved have been constrained using experimental information [28]. Studies have identified uncertainties in the rates of the ${ }^{18} \mathrm{~F}(\mathrm{p}, \alpha),{ }^{25} \mathrm{Al}(\mathrm{p}, \gamma)$ and ${ }^{30} \mathrm{P}(\mathrm{p}, \gamma)$ reactions as dominant contributors to remaining uncertainties in nova nucleosynthesis [21,29], and many recent measurements have focused on better determining these rates, including Refs. [30-39]. Additional theoretical studies are encouraged to examine the effects of recent measurements and to confirm the relative robustness of nova nucleosynthesis to nuclear physics uncertanities.

Nucleosynthesis in type I X-ray bursts, on the other hand, may eventually proceed toward the proton dripline through the $r p$ process and involve reactions on nuclei with considerably higher masses than considered in $\mathrm{CN}(A \approx 100)$. For this reason, explorations of the more exotic nature of the possible nucleosynthesis in these environments are forced to rely upon theoretical rates (from statistical model or shell model calculations) because of the lack of sufficient experimental information for most rates involved. While these theoretical rates are often stated to be reliable, on average, to a factor of $\approx 2$, significantly larger deviations have been observed when comparing (i) statistical model rates to experimental rates (up to a factor of 100 in some cases) and (ii) statistical model rates for a common reaction determined with different codes (up to an order of magnitude) [5]. Sensitivity studies using both one-zone methods [23,40] and 1D hydrodynamic models [41] have identified rates and masses required to better constrain predictions of nucleosynthesis in XRBs. These have helped to guide recent work (e.g., Refs. [42-48]), including the first measurements of the masses of ${ }^{65} \mathrm{As}$ and ${ }^{69} \mathrm{Br}[49,50]$. Knowledge of these masses is critical to understanding the $(\mathrm{p}, \gamma)-(\gamma, \mathrm{p})$ reaction rate equilibria that develop at the ${ }^{64} \mathrm{Ge}$ and ${ }^{68} \mathrm{Se}$ waiting points along the $r p$ process and the subsequent evolution of the abundance flow beyond these nuclei. The expected effect of the now-experimental $S_{p}\left({ }^{65} \mathrm{As}\right)$ and $S_{p}\left({ }^{69} \mathrm{Br}\right)$ values would be a reduction in the abundance flow beyond $A \approx 70$ relative to predictions using the estimated masses from Ref. [53]. This is supported by results shown in Fig. 2 from preliminary hydrodynamic calculations, although the influence of the new measurements on the yields is not large. Additional hydrodynamic models are needed to further explore the precise impact of these measurements in detail.

\section{Outlook}

When computationally feasible, modelers should work to evolve full multidimensional hydrodynamic model calculations of both classical nova explosions and type I X-ray bursts from early to late times. From the point of view of the associated nucleosynthesis, however, the limited nuclear networks coupled to these computationally-demanding simulations assures the endurance of results from 1D models for the time being. New sensitivity studies using both 1D hydrodynamic models and one-zone calculations are therefore needed to determine the role of uncertainties in the input nuclear physics on model predictions of nucleosynthesis and light curves. These studies should also focus upon resolving possible discrepancies observed between current XRB models, such as the impact of uncertainties in the ${ }^{15} \mathrm{O}(\alpha, \gamma)$ rate on predicted light curves $[6,54]$ or discerning the exact role of the metallicity of the accreted material on observable predictions [16,20]. Detailed 
evaluations using, e.g., sufficiently large numbers of bursts to ensure convergence of the calculations, would help to shed light on these issues. In the meantime, experimentalists should build further upon recent accomplishments to fully characterize the rates of the ${ }^{18} \mathrm{~F}(\mathrm{p}, \alpha),{ }^{25} \mathrm{Al}(\mathrm{p}, \gamma)$ and ${ }^{30} \mathrm{P}(\mathrm{p}, \gamma)$ reactions for nova explosions and determine the nuclear structure of e.g., ${ }^{62} \mathrm{Ge}$ and ${ }^{66} \mathrm{Se}$ above the proton threshold [23] for XRBs. Consistent treatments for calculating stellar weak rates for all isotopes in a typical XRB network are also needed.

New observatories, such as the recently-launched NuSTAR [55] and proposed LOFT [56] missions, hold promise for the clear detection of absorption features from XRBs. Such results could provide a much-needed direct constraint on nucleosynthesis in these environments. As well, prospects for the ejection of nuclear-processed material by radiation-driven winds from XRBs [57] still need to be evaluated in detail. For novae, UV observations had often been used in the past to determine abundances of ejected material (using, e.g., the now-defunct IUE [58]); the WSOUV mission (planned for launch in 2016) will help to further advance such studies [59]. High resolution X-ray spectra of nova ejecta have been obtained by e.g., Chandra and XMM-Newton, however improvements in expanding atmosphere models are necessary before abundances can be reliably extracted from these spectra $[60,61]$.

\section{Acknowledgments}

This work has been supported by the MICINN grant AYA2010-15685, the Government of Catalonia grant 2009SGR-1002, and the ESF EUROCORES Program EuroGENESIS through the MICINN grant EUI2009-04167. We appreciate comments from J. José and G. Sala on specific aspects of this brief review. 

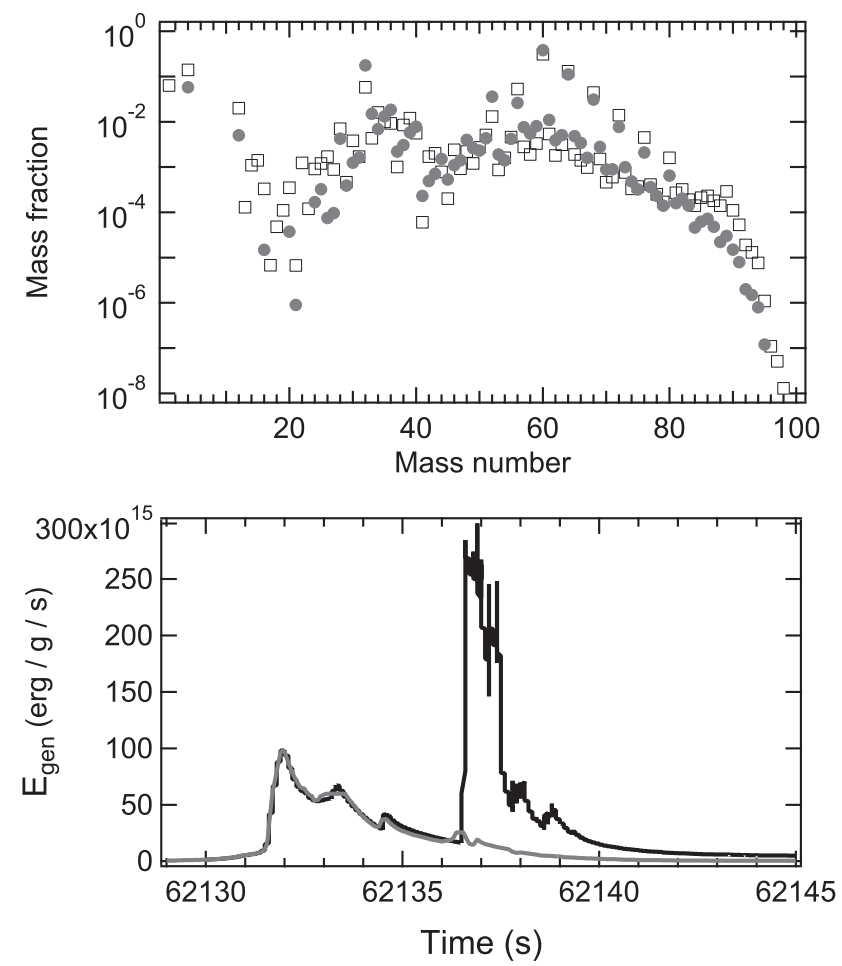

Figure 1: (Top) Final yields from a hydrodynamic XRB model (open squares, burst 3 from model 1 of Ref. [16]) and from a one-zone calculation (filled circles) using the hottest shell of the same model. (Bottom) Nuclear energy generation rate from the hottest shell of the same model, as found in the hydrodynamic calculation (black line) and from a one-zone calculation using temperature and density profiles from that shell (grey line).

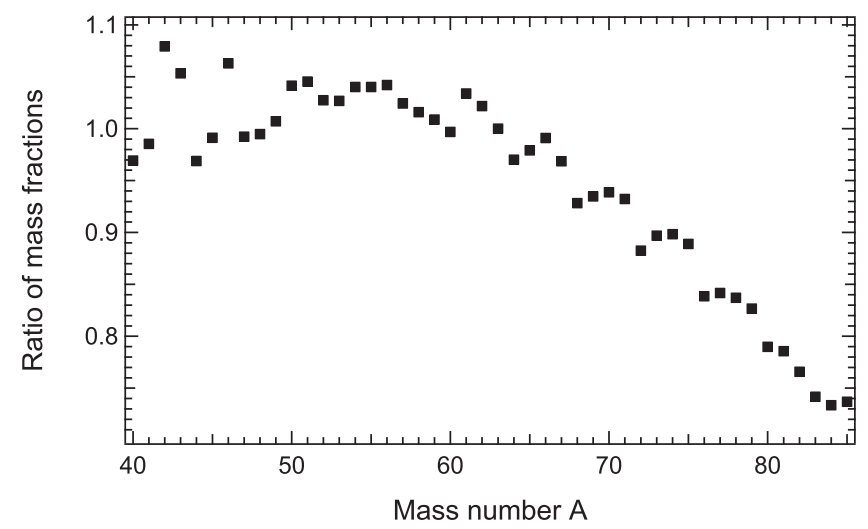

Figure 2: Ratio of final XRB mass fractions calculated assuming the recent experimental values for $S_{p}\left({ }^{65} \mathrm{As}\right)$ $[49,51]$ and $S_{p}\left({ }^{69} \mathrm{Br}\right)[50,52]$ to XRB mass fractions calculated assuming the corresponding estimated values from Ref. [53]. Mass fractions have been determined through otherwise identical hydrodynamic calculations (i.e., model 1 of Ref. [16]). 


\section{References}

[1] J. José, M. Hernanz, Nucleosynthesis in classical nova explosions, J. Phys. G: Nucl. Part. Phys. 34 (2007) R431.

[2] Classical Novae, eds. M. Bode, A. Evans, (Cambridge Univ. Press, Cambridge, 2nd ed., 2008).

[3] W.H.G. Lewin, J. van Paradijs, R.E. Taam, X-Ray Bursts, Space Sci. Rev. 62 (1993) 223.

[4] T. E. Strohmayer, L. Bildsten, New views of thermonuclear bursts, in Compact Stellar X-ray Sources, eds. W.H.G. Lewin, M. van der Klis, (Cambridge Astrophysics Series No. 39, Cambridge Univ. Press, Cambridge, 2006), p. 113.

[5] A. Parikh, J. José, G. Sala, C. Iliadis, Nucleosynthesis in Type I X-ray Bursts, Prog. Part. Nucl. Phys. (submitted, 2012).

[6] J.L. Fisker, W. Tan, J. Görres, M. Wiescher, R.L. Cooper, The ${ }^{15} O(\alpha, \gamma){ }^{19} N e$ Reaction Rate and the Stability of Thermonuclear Burning on Accreting Neutron Stars, Astrophys. J. 665 (2007) 637.

[7] A. Cumming, L. Bildsten, Carbon Flashes in the Heavy-Element Ocean on Accreting Neutron Stars, Astrophys. J. 559 (2001) L127.

[8] L. Keek, A. Heger, J.J.M. in't Zand, Superburst Models for Neutron Stars with Hydrogen- and Helium-rich Atmospheres, Astrophys. J. 752 (2012) 150.

[9] J.J.M. in't Zand, A. Cumming, M.V. van der Sluys, F. Verbunt, O.R. Pols, On the possibility of a helium white dwarf donor in the presumed ultracompact binary $2 S$ 0918-549, Astron. Astrophys. 441 (2005) 675.

[10] D.K. Galloway, A. Cumming, Helium-rich Thermonuclear Bursts and the Distance to the Accretion-powered Millisecond Pulsar SAX J1808.4-3658, Astrophys. J. 652 (2006) 559.

[11] E. Schatzman, Remarques sur le phénomène de novae, Ann. Astrophys. 12 (1949) 281.

[12] E. Schatzman, Remarques sur le phénomène de Nova. III. L'onde de choc dans la bombe à hydrogène, Ann. Astrophys. 13 (1950) 384.

[13] E. Schatzman, Remarques sur le phénomène de Nova: IV. L'onde de détonation due à l'isotope ${ }^{3} H e$, Ann. Astrophys. 14 (1951) 294.

[14] S.E. Woosley, R.E. Taam, Gamma-ray bursts from thermonuclear explosions on neutron stars, Nature 263 (1976) 101.

[15] L. Maraschi, A. Cavaliere, X-ray bursts of nuclear origin?, in Highlights in Astronomy IV, ed. E. Muller (Reidel, Dordrecht, 1977), p. 127.

[16] J. José, F. Moreno, A. Parikh, C. Iliadis, Hydrodynamic Models of Type I X-ray Bursts: Metallicity Effects, Astrophys. J. Suppl. 189 (2010) 204.

[17] O. Yaron, D. Prialnik, M.M. Shara, A. Kovetz, An Extended Grid of Nova Models. II. The Parameter Space of Nova Outbursts, Astrophys. J. 623 (2005) 398.

[18] J. José, E. García-Berro, M. Hernanz, P. Gil-Pons, The First Nova Explosions, Astrophys. J. 662 (2007) L103.

[19] S. Starrfield, F.X. Timmes, C. Iliadis, W.R. Hix, W.D. Arnett, C. Meakin, W.M. Sparks, Hydrodynamic Studies of the Evolution of Recurrent, Symbiotic and Dwarf Novae: the White Dwarf Components are Growing in Mass, Baltic Astr. 21 (2012) 76. 
[20] S.E. Woosley, A. Heger, A. Cumming, R.D. Hoffman, J. Pruet, T. Rauscher, J.L. Fisker, H. Schatz, B.A. Brown, M. Wiescher, Models for Type I X-Ray Bursts with Improved Nuclear Physics, Astrophys. J. Suppl. 151 (2004) 75.

[21] C. Iliadis, A. Champagne, J. José, S. Starrfield, P. Tupper, The Effects of Thermonuclear Reaction-Rate Variations on Nova Nucleosynthesis: A Sensitivity Study, Astrophys. J. Suppl. 142 (2002) 105.

[22] H. Schatz, A. Aprahamian, V. Barnard, L. Bildsten, A. Cumming, M. Ouellette, T. Rauscher, F.-K. Thielemann, M. Wiescher, End Point of the rp Process on Accreting Neutron Stars, Phys. Rev. Lett. 86 (2001) 3471.

[23] A. Parikh, J. José, F. Moreno, C. Iliadis, The Effects of Variations in Nuclear Processes on Type I X-Ray Burst Nucleosynthesis, Astrophys. J. Suppl. 178 (2008) 110.

[24] J. Casanova, J. José, E. García-Berro, S.N. Shore, A.C. Calder, Kelvin-Helmholtz instabilities as the source of inhomogeneous mixing in nova explosions, Nature 478 (2011) 490.

[25] C.M. Malone, A. Nonaka, A.S. Almgren, J.B. Bell, M. Zingale, Multidimensional Modeling of Type I $X$-ray Bursts. I. Two-dimensional Convection Prior to the Outburst of a Pure ${ }^{4} \mathrm{He}$ Accretor, Astrophys. J. 728 (2011) 118.

[26] J. José, M. Hernanz, Nucleosynthesis in Classical Novae: CO versus ONe White Dwarfs, Astrophys. J. 494 (1998) 680.

[27] J. L. Fisker, H. Schatz and F.-K. Thielemann, Explosive Hydrogen Burning during Type I X-Ray Bursts, Astrophys. J. Suppl. 174 (2008) 261.

[28] C. Iliadis, R. Longland, A.E. Champagne, A. Coc, R. Fitzgerald, Charged-particle thermonuclear reaction rates: II. Tables and graphs of reaction rates and probability density functions, Nucl. Phys. A841 (2010) 31.

[29] J. José, A. Coc, M. Hernanz, Synthesis of Intermediate-Mass Elements in Classical Novae: From Si to Ca, Astrophys. J. 560 (2001) 897.

[30] C.E. Beer et al., Direct measurement of the ${ }^{18} F(p, \alpha)^{15} O$ reaction at nova temperatures, Phys. Rev. C 83 (2011) 042801.

[31] A.S. Adekola et al., First proton-transfer study of ${ }^{18} \mathrm{~F}+\mathrm{p}$ resonances relevant for novae, Phys. Rev. C 83 (2011) 052801.

[32] A.S. Adekola et al., Single-nucleon transfer reactions on ${ }^{18} F$, Phys. Rev. C 84 (2011) 054611.

[33] A.S. Adekola et al., ${ }^{19}$ Ne levels studied with the ${ }^{18} F(d, n){ }^{19} N e\left({ }^{18} F+p\right)$ reaction, Phys. Rev. C 85 (2012) 037601.

[34] K.A. Chipps et al., The ${ }^{28} \mathrm{Si}(p, t)^{26} \mathrm{Si}^{*}(p)$ reaction and implications for the astrophysical ${ }^{25} \mathrm{Al}(p, \gamma)^{26} \mathrm{Si}$ reaction rate, Phys. Rev. C 82 (2010) 045803.

[35] A. Matic et al., High-precision $(p, t)$ reaction to determine ${ }^{25} A l(p, \gamma)^{26}$ Si reaction rates, Phys. Rev. C $82(2010) 025807$.

[36] J. Chen et al., ${ }^{26}$ Si excited states via one-neutron removal from a ${ }^{27}$ Si radioactive ion beam, Phys. Rev. C 85 (2012) 045809.

[37] J. Chen et al., Strong ${ }^{25} \mathrm{Al}+\mathrm{p}$ resonances via elastic proton scattering with a radioactive ${ }^{25} \mathrm{Al}$ beam, Phys. Rev. C 85 (2012) 015805. 
[38] A. Parikh et al., Improving the ${ }^{30} P(p, \gamma)^{31} S$ rate in oxygen-neon novae: Constraints on $J^{\pi}$ values for proton-threshold states in ${ }^{31} S$, Phys. Rev. C 83 (2011) 045806.

[39] D.T. Doherty et al., Key Resonances in the ${ }^{30} P(p, \gamma)^{31} S$ Gateway Reaction for the Production of Heavy Elements in ONe Novae, Phys. Rev. Lett. 108 (2012) 262502.

[40] A. Parikh, J. José, C. Iliadis, F. Moreno, T. Rauscher, Impact of uncertainties in reaction $Q$ values on nucleosynthesis in type I X-ray bursts, Phys. Rev. C 79 (2009) 045802.

[41] R. Cyburt et al., X-ray Burst Sensitivities, PoS(NIC-XII) 045.

[42] A. Banu et al., One-proton breakup of ${ }^{24} S i$ and the ${ }^{23} \mathrm{Al}(p, \gamma)^{24}$ Si reaction in type I $x$-ray bursts, Phys. Rev. C 86 (2012) 015806.

[43] C. Deibel et al., First measurement of the ${ }^{33} C l(p, \alpha)^{30} S$ reaction, Phys. Rev. C 84 (2011) 045802.

[44] C. Wrede et al., Properties of ${ }^{20} \mathrm{Na},{ }^{24} \mathrm{Al},{ }^{28} \mathrm{P},{ }^{32} \mathrm{Cl}$, and ${ }^{36} \mathrm{~K}$ for studies of explosive hydrogen burning, Phys. Rev. C 82 (2010) 035805.

[45] K. Setoodehnia et al., Structure of ${ }^{30} S$ with ${ }^{32} S(p, t)^{30} S$ and the thermonuclear ${ }^{29} P(p, \gamma)^{30} S$ reaction rate. Phys. Rev. C 82 (2010) 022801.

[46] J. Fallis et al., Mass measurements of isotopes of $\mathrm{Nb}, \mathrm{Mo}, \mathrm{Tc}, \mathrm{Ru}$, and Rh along the $\mathrm{vp}$ - and rp-process paths using the Canadian Penning trap mass spectrometer, Phys. Rev. C 84 (2011) 045807.

[47] A. Kankainen, Y.N. Novikov, H. Schatz, C. Weber, Mass measurements of neutron-deficient nuclei and their implications for astrophysics, Eur. Phys. J. A 48 (2012) 50.

[48] W.H. Zhang et al., Mass Measurements of the Neutron-Deficient ${ }^{41} \mathrm{Ti},{ }^{45} \mathrm{Cr},{ }^{49} \mathrm{Fe}$, and ${ }^{53} \mathrm{Ni}$ Nuclides: First Test of the Isobaric Multiplet Mass Equation in fp-Shell Nuclei, Phys. Rev. Lett. 109 (2012) 102501.

[49] X. L. Tu et al., Direct Mass Measurements of Short-Lived $A=2 Z-1$ Nuclides ${ }^{63} \mathrm{Ge},{ }^{65} \mathrm{As},{ }^{67} \mathrm{Se}$, and ${ }^{71} \mathrm{Kr}$ and Their Impact on Nucleosynthesis in the rp Process, Phys. Rev. Lett. 106 (2011) 112501.

[50] A. M. Rogers et al., Ground-State Proton Decay of ${ }^{69} \mathrm{Br}$ and Implications for the ${ }^{68}$ Se Astrophysical Rapid Proton-Capture Process Waiting Point, Phys. Rev. Lett. 106 (2011) 252503.

[51] P. Schury et al., Precision mass measurements of rare isotopes near $N=Z=33$ produced by fast beam fragmentation, Phys. Rev. C 75 (2007) 055801.

[52] J. Savory et al., rp Process and Masses of $N \approx Z \approx 34$ Nuclides, Phys. Rev. Lett. 102 (2009) 132501.

[53] G. Audi, A.H. Wapstra, C. Thibault, The AME2003 atomic mass evaluation. II. Tables, graphs and references, Nucl. Phys. A729 (2003) 337.

[54] B. Davids, R.H. Cyburt, J. José, S. Mythili, The Influence of Uncertainties in the ${ }^{15} O(\alpha, \gamma){ }^{19} \mathrm{Ne}$ Reaction Rate on Models of Type I X-Ray Bursts, Astrophys. J. 735 (2011) 40.

[55] F.A. Harrison et al., The Nuclear Spectroscopic Telescope Array (NuSTAR), Proc. SPIE 7732 (2010) $77320 \mathrm{~S}$.

[56] M. Feroci et al., LOFT: the Large Observatory For X-ray Timing, Proc. SPIE 8443 (2012) 84432D.

[57] J.J.M. in 't Zand, N.N. Weinberg, Evidence of heavy-element ashes in thermonuclear X-ray bursts with photospheric superexpansion, Astron. Astrophys. 520 (2010) A81.

[58] S.J. Austin, R.M. Wagner, S. Starrfield, S.N. Shore, G. Sonneborn, R. Bertram, V1974 Cygni 1992: Optical and Ultraviolet Evolution and Analysis, Astronom. J. 111 (1996) 869. 
[59] M. Hernanz et al., WSO/UV: World Space Observatory/Ultraviolet, AIP Conf. Proc. 637 (2002) 238.

[60] C. Pinto, J.-U. Ness, F. Verbunt, J.S. Kaastra, E. Costantini, R.G. Detmers, A phenomenological model for the X-ray spectrum of nova V2491 Cygni, Astron. Astrophys. 543 (2012) A134.

[61] D.R. van Rossum, A Public Set of Synthetic Spectra from Expanding Atmospheres for X-Ray Novae. I. Solar Abundances, Astrophys. J. 756 (2012) 43. 